\title{
Comportamiento productivo de pollos parrilleros alimentados con Moringa oleifera en Formosa, Argentina
}

\author{
Gómez, N.I. ; Rébak, G. ${ }^{2}$; Fernández, R. ${ }^{3}$; Sindik, M. ${ }^{3}$; Sanz, P. ${ }^{3}$ \\ ${ }^{1}$ Facultad de Producción y Ambiente, Universidad Nacional de Formosa, Laguna Blanca, Formosa. \\ Correspondencia: Uriburu 609, Formosa, Tel. +54370-4619109. E-mail: iuf@formosa.gov.ar. \\ ${ }^{2}$ Laboratorio de Tecnología de los Alimentos, ${ }^{3}$ Cátedra Producción de Aves, \\ Facultad de Ciencias Veterinarias UNNE, Corrientes, Argentina.
}

\begin{abstract}
Resumen
Gómez, N.I.; Rébak, G.; Fernández, R.; Sindik, M.; Sanz, P.: Comportamiento productivo de pollos parrilleros alimentados con Moringa oleifera en Formosa, Argentina. Rev. Vet. 27: 1, 7-10, 2016. Los altos costos de las materias primas utilizadas como fuente de proteínas, especialmente la soja, han llevado a la necesidad de investigar nuevas variantes para el reemplazo de este principio nutritivo en la elaboración de alimento aviar. El objetivo del trabajo fue estudiar el efecto de la incorporación de harina de hojas del árbol Moringa oleifera al alimento terminador sobre variables productivas, rendimientos de faena y de cortes comerciales, así como porcentaje de panículo adiposo en pollos parrilleros. En las dietas de prueba se incluyó 4\% (T1) y 8\% (T2) de hojas de moringa previamente deshidratadas, mientras que la dieta control (T0) fue elaborada en base a maíz, soja y una premezcla para alimento terminador. Bajo un modelo completamente aleatorizado, la significación estadística fue obtenida mediante ANOVA a una vía. No se observaron diferencias significativas $(\mathrm{p}<0,05)$ para las ganancias de peso $(1.631,67 \pm 55,41 \mathrm{~g}$ en $\mathrm{T} 0 ; 1.643,00 \pm 112,65 \mathrm{~g}$ en T1 y $1.562,67 \pm 26,76 \mathrm{~g}$ en T2). El consumo de alimento registró una diferencia estadísticamente significativa a favor de las dietas de prueba con valores de 4.043,33 $\pm 62,40 \mathrm{~g} ; 4.364,00 \pm 29,65$ g y 4.207,67 $\pm 115,00 \mathrm{~g}$ para T0, T1 y T2 respectivamente. Los rendimientos de carcasa fueron: $74,20 \%$ (T0), 73,93\% (T1) y 70,93\% (T2). Los rendimientos de pechuga fueron: $18,90 \%$, $18,41 \%$ y $19,88 \%$ para T0, T1 y T2 y los de pata-muslo de $35,47 \%, 34,99 \%$ y $37,19 \%$ para $\mathrm{T} 0$, T1 y T2. Se concluye que la harina de hojas de $M$. oleifera puede ser incluida hasta un $8 \%$ en la dieta de pollos parrilleros sin producir efectos adversos sobre la performance productiva, de faena y cortes.
\end{abstract}

Palabras clave: pollo, alimentación, fuente de proteínas, Moringa oleifera, eficiencia.

\begin{abstract}
Gómez, N.I.; Rébak, G.; Fernández, R.; Sindik, M.; Sanz, P.: Productive performance in bloiler chickens fed Moringa oleifera in Formosa, Argentina. Rev. Vet. 27: 1, 7-10, 2016. High costs of nutrients used as protein source -particularly soybean- has led to the need of new alternatives for replacement with less expensive foods for the preparation of poultry feeds. The aim of this study was to investigate the effect of the incorporation of Moringa oleifera flour in finisher diet for broilers on productive variables, carcass yield, meat cuts and percentage of panniculus adipose tissue. Trial diets included 4\% (T1) and 8\% (T2) dehydrated and powdered moringa leaves, whereas the control diet (T0) consisted of corn, soybean and finisher diet. Under a complete randomized model, statistical significance was obtained using one way ANOVA. No statistically significant differences $(\mathrm{p}<0.05)$ were observed for weight gain $(1631.67 \pm 55.41 \mathrm{~g}$ for T0; $1643.00 \pm 112.65 \mathrm{~g}$ for T1 y $1562.67 \pm 26.76 \mathrm{~g}$ for T2). Food consumption recorded a statistically significant difference considering tested diets, with values of $4043.33 \pm 62.40 \mathrm{~g} ; 4364.00 \pm 29.65 \mathrm{~g}$ and $4207.67 \pm 115.00 \mathrm{~g}$ for T0, T1 and T2, respectively. Carcass yields were $74.20 \%$ (T0), $73.93 \%$ (T1) and $70.93 \%$ (T2). Breast yields were $18.90 \%, 18.41 \%$ and $19.88 \%$ for $\mathrm{T} 0$, T1 and T2, respectively and $35.47 \%$ (T0), $34.99 \%$ (T1) and $37.19 \%$ (T2) for leg quarters. We conclude that $M$. oleifera meal can be included in
\end{abstract}


up to $8 \%$ of broiler diets without adverse effects on the productive, slaughter and meat cut performances.

Key words: chicken, feeding, source of proteins, Moringa oleifera, efficiency.

\section{INTRODUCCIÓN}

En los distintos sistemas de producción avícola la alimentación representa más del $70 \%$ de los costos operativos. Tanto a escala industrial como en la producción alternativa, los altos precios de las materias primas tradicionales, especialmente la soja, convencionalmente utilizada como fuente de proteína, han llevado a la necesidad de investigar nuevas alternativas para la obtención de este principio nutritivo, que representen opciones innovadoras y económicamente viables para la elaboración del alimento para las aves ${ }^{3,5,7}$.

Los estudios sobre las características químicas y composición centesimal de vegetales ricos en proteínas, entre los que se cuenta Moringa oleifera, auguran la eficiencia de esta materia prima, un producto atractivo por su valor nutricional y disponibilidad en el nordeste argentino, aunque aún no existen estudios biológicos y económicos que aseguren las ventajas de su empleo en aves.

El contenido proteico de las hojas de moringa es superior al $25 \%$, poseyendo además elevados valores de vitaminas A, B y C, así como minerales (particularmente hierro) y aminoácidos azufrados como metionina y cistina, posicionando a este vegetal como una materia prima de gran importancia en las dietas de ganado bovino, aves, peces y cerdos ${ }^{3}$. Además contiene muy bajos niveles de sustancias antinutritivas ${ }^{9}$.

En el presente trabajo se estudió el efecto de la incorporación de harina de moringa previamente secada y molida, en proporciones del 4 y $8 \%$ del alimento terminador sobre las variables productivas y rendimiento de canal, pechuga, muslos y panículo adiposo de pollos parrilleros machos híbridos Cobb 500.

\section{MATERIAL Y MÉTODOS}

El ensayo se llevó a cabo en mayo de 2014 en una granja avícola, ubicada en la localidad de Laguna Blanca, Departamento Pilcomayo, Provincia de Formosa (Argentina). Se utilizó un galpón semiabierto de $5 \mathrm{~m}$ x $16 \mathrm{~m}$, techo de cinc, sin cumbrera, paredes de mampostería, ventanas laterales y piso de cemento alisado. Se utilizó solamente un sector del galpón, equivalente a una dimensión de $30 \mathrm{~m}^{2}$, que fue dividido en $9 \mathrm{com}$ partimentos de $1,5 \mathrm{~m}^{2}$ cada uno. Las divisiones se realizaron con palmas "carandaa" (Copernicia alba) y mallas de media sombra. Se usó como cama cascarilla de arroz, de $7 \mathrm{~cm}$ de espesor, renovándose parcialmente en forma semanal.

Los sujetos experimentales fueron 100 pollos parrilleros híbridos, machos Cobb 500, de diez días de edad con peso medio de $90 \mathrm{~g}$, provistos por la Escuela de Enseñanza Agropecuaria $\mathrm{N}^{\circ} 8$, de General San Martín (Chaco, Argentina). El lote se crió a piso en forma conjunta, recibiendo las mismas condiciones ambientales y de manejo hasta el día 21, con un programa de luz continua durante la primera semana y luz natural en el periodo restante.

A los 21 días se dio inicio al ensayo, seleccionando al azar 90 ejemplares que se alojaron en 9 boxes a razón de 10 aves por compartimiento. El peso promedio de las aves fue $538,22 \pm 48,43 \mathrm{~g}$. En cada box el agua fue provista con bebederos de plato con recipiente invertido de 4 litros y el alimento con comederos tolva de $3 \mathrm{~kg}$ de capacidad. Durante el período del ensayo se registraron temperaturas máximas con un promedio de $34,6^{\circ} \mathrm{C}$ y mínimas de $14,8^{\circ} \mathrm{C}$; la humedad relativa ambiente osciló entre 59 y $86 \%$.

Se aplicó un diseño experimental completamente aleatorizado, en el cual cada box constituyó una unidad experimental y a su vez, una repetición simple de cada tratamiento. Para analizar las diferencias entre tratamientos (control y prueba con diferentes porcentajes de harina de moringa) se aplicó análisis de la variancia para un diseño a una vía completamente al azar, considerando límite un nivel de significancia del $5 \%{ }^{8}$.

La variable independiente estuvo constituida por tres tipos de dietas suministradas ad libitum desde el día 21 al 42 de vida. La dieta control se elaboró en base a maíz, soja y una premezcla para balanceado de parrillero; en las dietas de prueba $M$. oleifera reemplazó parcialmente a la harina de soja. Las hojas de moringa previamente fueron sometidas a deshidratación en un secadero manual a temperatura de $60^{\circ} \mathrm{C}$ durante 4 días y luego molidas a un tamaño de $1 \mathrm{~mm}$. Las muestras de los ingredientes fueron analizadas mediante el procedimiento de la AOAC (1990) ${ }^{1}$. La composición proximal de la harina de $M$. oleifera reveló contenidos de 23,69\% de proteína bruta, $4,42 \%$ extracto etéreo, $4,45 \%$ de fibra bruta y un valor de energía metabolizable de 3.390 $\mathrm{kcal} / \mathrm{kg}$. La composición centesimal de los alimentos se expone en tabla 1.

Durante el ensayo se registraron las siguientes variables dependientes: (1) ganancia de peso (g), obtenida por diferencia entre los promedios de peso corporal final e inicial en cada box; (2) consumo de alimento (g): total de alimento consumido durante el ensayo en cada box divido la cantidad de aves; y (3) conversión alimenticia $(\mathrm{g} / \mathrm{g})$ : relación entre alimento consumido y ganancia de peso.

Las aves fueron sacrificadas por yugulación previa insensibilización ${ }^{4}$, seguida por la evisceración, lavado y enfriado de la canal con agua clorinada y hielo. 
Tabla 1. Composición y análisis proximal de las dietas suministradas.

\begin{tabular}{lccc}
\hline composición & $\begin{array}{c}\text { alimento } \\
\text { control (T0) }\end{array}$ & $\begin{array}{c}\text { moringa 4\% } \\
(\mathrm{T} 1)\end{array}$ & $\begin{array}{c}\text { moringa 8\% } \\
\text { (T2) }\end{array}$ \\
\hline composición porcentual & & & \\
maíz (\%) & 65 & 65 & 65 \\
soja (\%) & 30 & 26 & 22 \\
moringa (\%) & 0 & 4 & 8 \\
premezcla (\%) & 5 & 5 & 5 \\
\hline composición centesimal & & & \\
materia seca & 93,17 & 93,04 & 93,37 \\
proteína bruta & 15,10 & 16,28 & 15,14 \\
extracto etéreo & 6,33 & 6,75 & 6,79 \\
fibra bruta & 3,08 & 3,43 & 5,18 \\
cenizas & 6,47 & 6,53 & 6,95 \\
energía met.(kcal $/ \mathrm{kg})$ & 3,53 & 3,50 & 3,42 \\
\hline
\end{tabular}

Luego de escurridas y enfriadas se procedió al troceo de acuerdo a presentaciones comerciales, para obtener las variables siguientes: (1) rendimiento de canal (\%): se obtuvo dividiendo el peso de la canal (sin cabeza, patas ni vísceras) por el peso corporal y luego multiplicando por 100; (2) rendimiento de pechuga (\%): luego de separar y pesar la pechuga sin hueso, se obtuvo su porcentaje con respecto al peso limpio; y (3) rendimiento de pata muslo (\%): luego de separar y pesar la pata muslo con hueso, se obtuvo su porcentaje con respecto al peso limpio.

Se calcularon los estadísticos descriptivos media aritmética, desvío estándar, coeficiente de variación, mínimo y máximo de cada una de las variables dependientes. La distribución de todas las variables se constató mediante el método de Wilk Shapiro modificado y la homogeneidad de las varianzas mediante la técnica de Levene. El análisis comparativo se realizó mediante ANOVA.

\section{RESULTADOS}

En la tabla 2 se exhiben los valores de la estadística descriptiva en pollos parrilleros híbridos al final del período de estudio.

En las tablas 3 y 4 se exponen los resultados obtenidos para las variables productivas y rendimiento de cortes comerciales en pollos parrilleros híbridos según tratamientos.

\section{DISCUSIÓN}

El incremento constante en la demanda de materias primas que cubran los requerimientos alimentarios de las aves, ha impulsado investigaciones sobre la utilización de fuentes alternativas de proteínas de alta

Tabla 2. Valores obtenidos para las variables productivas y cortes comerciales.

\begin{tabular}{lccccc}
\hline variable & media & DE & CV & mínimo & máximo \\
\hline peso corporal a los 42 días $(\mathrm{g})$ & $2.150,67$ & 98,29 & 4,57 & $2.024,00$ & $2.306,00$ \\
ganancia peso total $(\mathrm{g})$ & $1.612,44$ & 74,41 & 4,61 & $1.517,00$ & $1.734,00$ \\
consumo de alimento $(\mathrm{g})$ & $4.205,00$ & 154,22 & 3,67 & $3.974,00$ & $4.397,00$ \\
conversión alimenticia (g/g) & 2,61 & 0,16 & 6,17 & 2,35 & 2,90 \\
rendimiento de canal (\%) & 73,02 & 2,95 & 4,03 & 65,74 & 75,26 \\
rendimiento de pechuga (\%) & 19,06 & 1,21 & 6,33 & 17,43 & 21,32 \\
rendimiento de pata muslo (\%) & 35,89 & 2,67 & 7,44 & 33,23 & 41,42 \\
panículo adiposo (\%) & 2,00 & 0,45 & 22,54 & 1,14 & 2,59 \\
\hline
\end{tabular}

media: media aritmética, DE: desvío estándar, CV: coeficiente de variación.

Tabla 3. Parámetros productivos registrados en pollos parrilleros.

\begin{tabular}{lccccccccc}
\hline & \multicolumn{2}{c}{ control } & \multicolumn{2}{c}{ moringa $4 \%$} & \multicolumn{2}{c}{ moringa 8\% } & F & p valor \\
\cline { 2 - 7 } & media & EE & media & EE & media & EE & & 0,41 \\
ganancia de peso $(\mathrm{g})$ & $1.643,00$ & 31,99 & $1.631,07$ & 65,04 & $1.562,67$ & 15,45 & 1,03 & 0,006 \\
consumo de alimento $(\mathrm{g})$ & $4.043,33$ & 36,03 & $4.364,00$ & 17,06 & $4.207,67$ & 66,42 & 12,85 & 0,006 \\
conversión alimenticia $(\mathrm{g} / \mathrm{g})$ & 2,48 & 0,07 & 2,67 & 0,12 & 2,69 & 0,05 & 1,81 & 0,24 \\
\hline
\end{tabular}

EE: error estándar, F: distribución.

Tabla 4. Rendimiento de canal y cortes comerciales.

\begin{tabular}{|c|c|c|c|c|c|c|c|c|}
\hline & \multicolumn{2}{|c|}{ control } & \multicolumn{2}{|c|}{ moringa $4 \%$} & \multicolumn{2}{|c|}{ moringa $8 \%$} & \multirow[b]{2}{*}{$\mathrm{F}$} & \multirow[b]{2}{*}{$\mathrm{p}$ valor } \\
\hline & media & $\mathrm{EE}$ & media & $\mathrm{EE}$ & media & $\mathrm{EE}$ & & \\
\hline rendimiento de canal (\%) & 74,20 & 0,82 & 73,93 & 0,32 & 70,93 & 2,74 & 1,19 & 0,36 \\
\hline rendimiento de pechuga $(\%)$ & 18,90 & 0,63 & 18,41 & 0,50 & 19,88 & 0,85 & 1,23 & 0,35 \\
\hline rendimiento pata muslo (\%) & 35,47 & 1,32 & 34,99 & 0,90 & 37,19 & 2,37 & 0,49 & 0,63 \\
\hline panículo adiposo $(\%)$ & 1,91 & 0,17 & 2,25 & 0,13 & 1,84 & 0,42 & 0,64 & 0,55 \\
\hline
\end{tabular}

EE: error estándar, F: distribución. 
calidad nutricional y bajo costo, orientando la mayor parte de los estudios exploratorios hacia especies vegetales localmente adaptadas. Entre ellas se destaca M. oleifera por su contenido de nutrientes esenciales, rápido crecimiento y simplicidad de las técnicas para su procesamiento, aunque la presencia de factores antinutricionales podría constituir una limitante para su utilización en aves.

En el presente estudio, la incorporación de harina de moringa produjo una diferencia significativa en el consumo de alimento a favor de las aves que recibieron moringa en su dieta, en tanto que la ganancia de peso y la conversión alimenticia fueron semejantes en los tres grupos experimentales.

Los resultados de las investigaciones sobre el efecto de la inclusión de M. oleifera sobre el consumo de alimento y otros parámetros productivos de las aves no son concluyentes, existiendo discrepancias relativas al efecto sobre el consumo de alimento, que en parte se deben a los niveles de inclusión aplicados, aunque se ha señalado la menor palatabilidad de esta materia prima como causa del menor consumo ${ }^{2}$.

Otros investigadores estudiaron los efectos del reemplazo de la soja por M. oleifera en pollos para carne con cinco niveles de inclusión de la harina en las dietas formuladas, una dieta control $(0 \%$ de harina de moringa) y niveles de reemplazo crecientes desde el 25 hasta el $100 \%$ de la soja. Si bien no se observaron diferencias significativas en la cantidad de alimento consumido, la conversión alimenticia empeoró proporcionalmente en las dietas con mayor inclusión de moringa debido a variaciones en el peso corporal ${ }^{3}$.

En otro estudio sobre reemplazo de soja por moringa se incluyeron dietas conteniendo $0,5,10,15$ y $20 \%$ de harina de $M$. oleifera, observándose una declinación de todos los índices técnicos de la producción en forma proporcional al nivel de inclusión del material de sustitución ${ }^{9}$. Los resultados obtenidos en el presente estudio permiten afirmar que, aunque la harina de hojas de moringa contiene niveles moderados a altos de proteína, su contenido de fibra relativamente alto puede provocar un aumento del consumo de alimento, afectando su utilidad como un recurso de alimentación alternativa para las especies aviares.

La inclusión de moringa en las dietas de prueba no produjo efectos sobre la composición corporal, incluyendo el rendimiento de la canal, cortes comerciales y contenido de grasa abdominal ${ }^{6}$. Otros investigadores evaluaron el efecto de cinco niveles de reemplazo $(0$, $5,10,15$ y $20 \%$ ) sobre los indicadores técnicos de la producción y rendimiento de la canal en dietas isocalóricas e isonitrogenadas y cortes comerciales en pollos parrilleros de ciclo largo (56 días), en un diseño factorial incluyendo el sexo como fuente de variación. El estudio demostró que el peso de faena, el rendimiento de la canal y de los cortes de valor comercial fueron más bajos en las aves que recibieron alimento conteniendo harina de moringa, señalando a la caída del consumo de alimento como factor determinante para la reduc- ción del rendimiento en los animales que recibieron la dieta prueba ${ }^{9}$.

En otro estudio se alimentaron pollos de crecimiento lento en período de terminación con dietas que incluyeron $2,5 \%, 5 \%$ y $10 \%$ de harina de hojas de $M$. oleifera observando diferencias significativas por sexo y también en relación con la harina puesta a prueba, concluyéndose que pollos de crecimiento lento asimilan adecuadamente este tipo de materia prima, conformando la factibilidad de usarla sin producir efectos perjudiciales ${ }^{7}$.

Se concluye que la harina de hojas de M. oleifera puede ser incluida en forma segura hasta en un $8 \%$ de las dietas para pollos parrilleros sin producir efectos adversos sobre la performance productiva ni sobre el rendimiento de la carcasa y cortes de valor comercial. Si bien se observa un aumento en el consumo de alimento en aves cuyas dietas incluyeron moringa, la misma no produce consecuencias sobre el peso corporal y el rendimiento, lo que permite aseverar que el perfil de nutrientes presente en esta materia prima y su asimilación en pollos parrilleros se adecua a sus requerimientos nutricionales.

Agradecimiento. A la Escuela de Enseñanza Agropecuaria $\mathrm{N}^{\circ} 8$ de General San Martín (Chaco, Argentina) por proveer los pollitos para el ensayo.

\section{REFERENCIAS}

1. AOAC. 1990. Official methods of analysis, $15^{\text {th }}$ ed., Publ. Association of Official Analytical Chemists, Virginia, USA.

2. Banjo OS. 2012. Growth and performance as affected by inclusion of Moringa oleifera leaf meal in broiler chicks diet. $J$ Biol 2: 35-38.

3. Gadzirayi CT, Masamha B, Mupangwa JF, Washaya S. 2012. Performance of broiler chickens fed on mature Moringa oleifera leaf meal as a protein supplement to soybean meal. Int J Poultry Sci 11: 5-10.

4. Mota Rojas D. et al. 2008. Welfare at slaughter of broiler chickens: a review. Int J Poultry Sci 7: 1-5.

5. Nouman W, Basra S, Siddiqui M, Yasmeen A, Gull T, Alcayde M. 2014. Potential of Moringa oleifera as livestock fodder crop: a review. Turk J Agric For 38: 1-14.

6. Ochi EB, Elbushra ME, Fatur M, Abubakr OI, Hafiz A. 2015. Effect of moringa (Moringa oleifera Lam) seeds on the performance and carcass characteristics of broiler chickens. J Nat Sci Res 5: 66-73.

7. Sebola NA, Mlambo V, Mokoboki HK, Muchenje V. 2015. Growth performance and carcass characteristics of three chicken strains in response to incremental levels of dietary Moringa oleifera leaf meal. Livestock Sci 178: 202208.

8. Steel R., Torrie J. 1988. Bioestadística: principios y procedimientos, McGraw-Hill/Interamericana, México, 622 p.

9. Tesfaye E, Animut G, Urge M, Dessie T. 2013. Moringa oleifera leaf meal as an alternative protein feed ingredient in broiler ration. Int J Poultry Sci 12: 289-297. 